\title{
Identifying the leukocyte uptake pattern of inflammation imaging agents: Current limitations and potential impact
}

\author{
Laurent Riou, PhD, ${ }^{\text {a Jakub Toczek, PhD, }}$, Alexis Broisat, PhD, ${ }^{\text {a }}$ \\ Catherine Ghezzi, PhD, ${ }^{a}$ and Loïc Djaileb, $M^{a, c}$ \\ a Laboratoire Radiopharmaceutiques Biocliniques, Faculté de Médecine de Grenoble, UMR UGA \\ - INSERM U1039, Grenoble, France \\ b Cardiovascular Molecular Imaging Laboratory, Section of Cardiovascular Medicine and Yale \\ Cardiovascular Research Center, Yale University School of Medicine, New Haven, CT \\ c Nuclear Medicine Department, Grenoble Alpes University Hospital, Grenoble, France
}

Received Nov 22, 2019; accepted Nov 22, 2019

doi: $10.1007 /$ s12350-019-01979-3

\section{See related article, pp. 1636-1645}

Inflammation plays an essential role in a number of cardiovascular diseases. ${ }^{1}$ The inflammatory process is characterized by tissue infiltration of leukocytes in response to chemoattractant signals. Leukocyte profile can differ for various pathologies at different stages of the disease, with differential consequences on disease progression and outcome. In particular, the persistence of a sustained inflammatory process or the resolution of inflammation and return to tissue homeostasis are driven by various cell types under differential stimuli and involve complex interplay between innate and adaptive immunity. Macrophages are believed to be of crucial importance in this process, with the ability to acquire distinct functional phenotypes: a pro-inflammatory M1 polarization and an alternative M2 polarization, mediating the resolution of inflammation. While the dichotomized view of macrophage polarization does not accurately represent the full spectrum of polarization observed in vivo, ${ }^{2}$ numerous studies relied on this simplified view and the findings remains largely relevant. For example, in atherosclerosis, the presence of M1

Reprint requests: Laurent Riou, $\mathrm{PhD}$, Laboratoire Radiopharmaceutiques Biocliniques, Faculté de Médecine de Grenoble, UMR UGA INSERM U1039, Grenoble, France; Laurent.Riou@univ-grenoblealpes.fr

J Nucl Cardiol 2021;28:1646-8.

1071-3581/\$34.00

Copyright (C) 2019 American Society of Nuclear Cardiology. macrophages has been associated with symptomatic and vulnerable plaques whereas M2 macrophages were shown to be abundant in stable, asymptomatic lesions, ${ }^{3}$ while in post-myocardial infarction, the M1 macrophage-driven non-resolving inflammatory process is associated with adverse tissue remodeling. ${ }^{4}$ Tissue macrophages derive in part from circulating monocytes, which represent a heterogenous population with pro- or anti-inflammatory properties. ${ }^{5}$ The differentiation into macrophages and polarization in the tissue are influenced by the local environment, particularly the cytokines profile associated with the specific $\mathrm{T}$ helper cells subpopulation. ${ }^{2}$ While typically less abundant compared to macrophages, helper $\mathrm{T}$ cells and regulatory $\mathrm{T}$ cells are recognized for their functional role in various chronic inflammatory diseases. ${ }^{6}$ In most cases, the involvement of other cells from adaptive immunity and its significance is less clear. ${ }^{1}$ Neutrophils, the most abundant leukocyte subtype, are classically associated with the initial phase of the inflammatory response ${ }^{4}$ but were shown to contribute to chronic inflammation as well. $^{7}$

The study by Borchert et al. in the present issue of the Journal of Nuclear Cardiology ${ }^{8}$ systematically evaluates the in vitro cellular uptake of six inflammation imaging agents on four leukocyte subpopulations isolated from whole human blood, differentially polarized macrophages, and cardiac cells. The evaluated tracers include ${ }^{18}$ F-Fluorodesoxyglucose (FDG), ${ }^{11 / 14} \mathrm{C}-\mathrm{me}$ thionine (MET), and ${ }^{18}$ F-fluorethyltyrosine (FET) as tracers of glucose or amino acid metabolism, and ${ }^{68} \mathrm{Ga}-$ DOTATATE, ${ }^{68}$ Ga-pentixafor, and ${ }^{18}$ F-flutriciclamide (GE180) as molecular imaging agents of inflammation targeting respectively SSTR2, CXCR4, and TSPO. The 
motivation of the study was to better define the in vitro cellular uptake profile of the tracers with the ultimate perspective that such insights into their detailed behavior might eventually provide the clinician with tools for a more relevant use of tracers. The systematic methodology chosen by the authors provides an easy and reproducible test allowing the reliable determination of the leukocyte uptake pattern of tracers.

The results indicated that FDG, MET, and GE180 displayed higher uptake in pro-inflammatory M1 macrophages whereas FET, DOTATATE, and pentixafor displayed overall homogenous uptake in all cell types with subtle yet statistically significant variations that are discussed by the authors. The observation of enhanced accumulation of FDG in M1 (or lipopolysaccharide) polarized macrophages is qualitatively similar to previously reported results. ${ }^{9}$ The differential uptake with unpolarized or alternatively polarized macrophages is highly variable between studies, stressing the sensitivity to experimental conditions. Additionally, several factors affecting FDG uptake have been described, such as hypoxia $^{10}$ or the presence of modified low-density lipoproteins. ${ }^{11}$ For those reasons, it seems unclear to what degree these findings can be extrapolated to various pathophysiological conditions. MET data nicely recapitulate earlier findings, obtained in similar studies. $^{12}$ In contrast to extensively studied FDG, little is known about the determinants of MET uptake. As a metabolic tracer, MET is potentially susceptible to similar modulation as FDG. MET uptake in M1 macrophages should be confirmed in a broader range of conditions relevant to in vivo pathophysiological settings, to enhance the confidence in the transitional value of these findings. Reports on the level of TSPO expression in relation to polarization profile has been inconsistent. ${ }^{13}$ While the expression of TSPO was not directly evaluated, the observation of higher uptake of GE180 in M1 macrophage in this study is consistent with the dominant view associating TSPO expression to a pro-inflammatory state. ${ }^{13}$ More generally, the evaluation of the expression level of SSTR2, CXCR4, and TSPO in the different cell types and its correlation to the uptake of the respective molecular imaging agent would have been an informative step of internal validation. Of note, FDG uptake in cardiomyocytes cultured in the presence of glucose was comparable to the level of M1 polarized macrophages. FDG uptake in cardiac fibroblasts was higher than any of the tested leucocyte population except for M1 macrophages. This highlights the known limitation of FDG for cardiac inflammation imaging. Interestingly, GE180 also displayed high cardiomyocyte uptake. As noted by the authors, the elevated uptake of a TSPO targeted tracer is not surprising considering the high content of mitochondria in the cardiac muscle. This could however be a pitfall for the imaging of inflammation using TSPO targeted tracers in a context requiring low myocardial uptake.

An additional observation is that the overall absolute cellular uptake of FDG and GE180 was 10 to 100fold higher than that observed for MET, FET, pentixafor, and DOTATATE. The cardiomyocytes and cardiac fibroblasts uptake of both tracers was of the same order of magnitude than that observed in blood-derived leucocytes and macrophage cell lines. This assessment was performed in standardized conditions; as a corollary, those conditions were not optimized for the different tracers and cell types. A comparable initial activity was used for all tracers, corresponding approximatively to the initial tracer concentration in blood, and the same incubation time was used in all cases. Specific activity or molar concentration was not detailed, but the amount of tracer most likely exceeded the values expected in the targeted tissues, especially compared to the late phase of tracer distribution. Nevertheless, there are in vivo data attesting of the translational value of those findings. Indeed, the difference in FDG and pentixafor uptake is concordant with the in vivo signal observed in a preclinical study, which showed that the pentixafor signal in infarcted myocardium was an order of magnitude lower that non-suppressed FDG cardiac uptake for a comparable injected dose. ${ }^{14}$ Radiotracers can present a wide range of tissue uptake. The performance of an imaging agent is mainly related to the signal-to-noise ratio obtained in the targeted tissue, and not to the absolute uptake level, providing that it substantially exceeds the detection limit of the imaging system.

The routine clinical imaging of cardiac infiltrative inflammatory diseases is mainly performed in the setting of infective endocarditis, cardiac sarcoidosis, and large vessel vasculitis and is limited to the use of autologous radiolabeled leukocytes and FDG. ${ }^{15-17}$ FDG PET imaging is widely used in clinical trials to evaluate tissue inflammation in a broad range of pathological conditions. As a non-metabolizable glucose analogue, FDG accumulates in cells with high glycolytic activities, including inflammatory cells. ${ }^{18}$ However, FDG presents with elevated myocardial uptake which reliable suppression is difficult to reach despite proper patient preparation prior to imaging. ${ }^{19}$ This is a well-documented major limitation of FDG for the imaging of cardiac or coronary inflammatory. In the recent years, novel imaging agents targeting the inflammatory process have been introduced, mostly in preclinical settings. ${ }^{20}$ While the cellular uptake mechanisms of those tracers are well defined, comparative data on uptake in different population of leucocytes and macrophage phenotype are sparse despite being a likely essential parameter of tracer development. In this regard, the study by Borchert 
et al. is an important step in the assessment of the potential of existing or novel tracers. Indeed, tracer pharmacokinetics directly determine tracer uptake at the site of interest. In this regard, tracer distribution including binding to blood cells and plasma proteins, tracer extraction by tissues, and tracer excretion and related blood clearance are all essential considerations. All those elements can be assessed experimentally, with compartmental modeling and kinetic analysis being mostly useful. Ultimately, favorable pharmacokinetics and desirable cell-specific uptake profile are required for a successful imaging agent. The study by Borchert $e t$ al. contributes to improve our understanding of the latter component, paving the way to more refined procedures to assess inflammation in vivo.

\section{Disclosure}

Laurent Riou, Jakub Toczek, Alexis Broisat, Catherine Ghezzi and Loï Djaileb have no conflict of interest to disclose.

\section{References}

1. Ruparelia N, Chai JT, Fisher EA, Choudhury RP. Inflammatory processes in cardiovascular disease: A route to targeted therapies. Nat Rev Cardiol 2017;14:314.

2. Murray PJ. Macrophage Polarization. Annu Rev Physiol 2017;79:541-66.

3. Chinetti-Gbaguidi G, Colin S, Staels B. Macrophage subsets in atherosclerosis. Nat Rev Cardiol 2015;12:10-7.

4. Ong SB, Hernandez-Resendiz S, Crespo-Avilan GE, Mukhametshina RT, Kwek XY, Cabrera-Fuentes HA, et al. Inflammation following acute myocardial infarction: Multiple players, dynamic roles, and novel therapeutic opportunities. Pharmacol Ther 2018;186:73-87

5. Italiani $\mathrm{P}$, Boraschi D. From monocytes to M1/M2 macrophages: Phenotypical vs. functional differentiation. Front Immunol 2014;5:514

6. Tabas I, Lichtman AH. Monocyte-macrophages and T cells in atherosclerosis. Immunity 2017;47:621-34.

7. Soehnlein O, Steffens S, Hidalgo A, Weber C. Neutrophils as protagonists and targets in chronic inflammation. Nat Rev Immunol 2017;17:248-61.

8. Borchert T, Beitar L, Langer LBN, Polyak A, Wester HJ, Ross TL, et al. Dissecting the target leukocyte subpopulations of clinically relevant inflammation radiopharmaceuticals. J Nucl Cardiol 2019. https://doi.org/10.1007/s12350-019-01929-z.

9. Tavakoli S, Zamora D, Ullevig S, Asmis R. Bioenergetic profiles diverge during macrophage polarization: Implications for the interpretation of $18 \mathrm{~F}-\mathrm{FDG}$ PET imaging of atherosclerosis. J Nucl Med 2013;54:1661-7.
10. Folco EJ, Sukhova GK, Quillard T, Libby P. Moderate hypoxia potentiates interleukin-1 beta production in activated human macrophages. Circ Res 2014;115:875-83.

11. Lee SJ, Quach CHT, Jung KH, Paik TY, Lee JH, Park JW, et al. Oxidized low-density lipoprotein stimulates macrophage F-18FDG uptake via hypoxia-inducible factor-1 alpha activation through Nox2-dependent reactive oxygen species generation. J Nucl Med 2014;55:1699-705.

12. Thackeray JT, Bankstahl JP, Wang Y, Wollert KC, Bengel FM. Targeting amino acid metabolism for molecular imaging of inflammation early after myocardial infarction. Theranostics 2016;6:1768-79.

13. Airas L, Nylund M, Rissanen E. Evaluation of microglial activation in multiple sclerosis patients using positron emission tomography. Front Neurol 2018;9:181.

14. Thackeray JT, Derlin T, Haghikia A, Napp LC, Wang Y, Ross TL, et al. Molecular imaging of the chemokine receptor CXCR4 after acute myocardial infarction. JACC Cardiovasc Imaging 2015;8:1417-26.

15. Habib G, Lancellotti P, Antunes MJ, Bongiorni MG, Casalta JP, Del Zotti F, et al. 2015 ESC Guidelines for the management of infective endocarditis: The Task Force for the Management of Infective Endocarditis of the European Society of Cardiology (ESC). Endorsed by: European Association for Cardio-Thoracic Surgery (EACTS), the European Association of Nuclear Medicine (EANM). Eur Heart J 2015;36:3075-128.

16. Slart R, Glaudemans A, Lancellotti P, Hyafil F, Blankstein R, Schwartz RG, et al. A joint procedural position statement on imaging in cardiac sarcoidosis: From the Cardiovascular and Inflammation \& Infection Committees of the European Association of Nuclear Medicine, the European Association of Cardiovascular Imaging, and the American Society of Nuclear Cardiology. J Nucl Cardiol 2018;25:298-319.

17. Slart RHJA. Writing group, Reviewer group, Members of EANM Cardiovascular, Members of EANM Infection \& Inflammation, Members of Committees, SNMMI Cardiovascular, Members of Council, PET Interest Group, Members of ASNC, EANM Committee Coordinator \& FDG-PET/CT(A) imaging in large vessel vasculitis and polymyalgia rheumatica: Joint procedural recommendation of the EANM, SNMMI, and the PET Interest Group (PIG), and endorsed by the ASNC. Eur J Nucl Med Mol Imaging 2018:45:1250-69.

18. Jamar F, Buscombe J, Chiti A, Christian PE, Delbeke D, Donohoe $\mathrm{KJ}$, et al. EANM/SNMMI guideline for F-18-FDG use in inflammation and infection. J Nucl Med 2013;54:647-58.

19. Osborne MT, Hulten EA, Murthy VL, Skali H, Taqueti VR, Dorbala S, et al. Patient preparation for cardiac fluorine-18 fluorodeoxyglucose positron emission tomography imaging of inflammation. J Nucl Cardiol 2017;24:86-99.

20. Vigne J, Thackeray J, Essers J, Makowski M, Varasteh Z, Curaj A, et al. Current and emerging preclinical approaches for imagingbased characterization of atherosclerosis. Mol Imaging Biol 2018;20:869-87.

Publisher's Note Springer Nature remains neutral with regard to jurisdictional claims in published maps and institutional affiliations. 